Send your letters to the Editor,

British Dental Journal,

64 Wimpole Street

London

W1G 8YS

Email bdj@bda.org

Priority will be given to letters less than 500 words long.

Authors must sign the letter, which

may be edited for reasons of space.

\section{CARE HOME HEALTH}

Sir, we are writing in reply to our Newcastle colleague providing domiciliary care for residents in care homes (Care for all; BDJ 2009; 206: 396). In Sheffield we see the same problems described, of poor oral hygiene in older adults in residential care. The residents are often unable to say what they need in terms of their care; many are unable to maintain oral health by themselves.

Residential Oral Care in Sheffield (ROCS) has been developed over the last few years in an attempt to address the problem. Supported by NHS Sheffield, ROCS utilises a number of GDPs and their practice teams, who are familiar with working in domiciliary settings. The GDPs provide annual oral health screenings and dental treatment, with specialist support from an SDO in the CDS. We have tried to tackle the poor oral health of residents by attempting to raise the awareness and educate the carers. We are doing this in conjunction with our local Oral Health Promotion Unit and the School of Dental Hygiene and Therapy at the University of Sheffield. We utilise Dental Hygiene and Therapy students to deliver a standardised oral health education programme for carers working in the care homes. This provides students with a unique insight and experience of oral health care, that they would not otherwise see. It also provides ROCS with the means to deliver simple oral health education to greater numbers of carers and homes in a more cost effective way than via the GDPs alone.

The poor oral health seen amongst residents in care homes and the difficulties in providing dental treatment can seem overwhelming. Prevention is the key. The ROCS team meets regularly, and we are continually reviewing prevention, education and clinical practice. Our ROCS team includes: GDPs with their dental nurses and DCPs, an SDO, Oral Health Promotion, Hygiene and Therapy, NHS Commissioners, and Dental Public Health. We also are looking at ways to liaise with other healthcare providers that are involved with these residents, to improve oral health.

With ROCS we feel we are making some improvement. As we visit the homes on a regular basis, we develop a rapport with the care home managers and care staff, gradually increasing the general oral health awareness amongst staff in the home. When it works well, the care home staff become part of the team too, working with us, helping maintain the oral health of the residents.

This sort of care does not come cheap and it certainly does not lend itself to a strict UDA system. It requires flexibility and understanding on behalf of the local commissioners.

We agree that there is a universal problem of poor oral health in older adults in residential care, and like our Newcastle colleague we would welcome any further input that may help improve their oral health.

K. Lines, G. Heyes ROCS Sheffield DOI: 10.1038/sj.bdj.2009.671

\section{A UNIVERSAL PROBLEM}

Sir, I am writing in response to the letter entitled Care for all (BDJ 2009; 206: 396) which highlighted the deficient level of oral care provided in residential care homes. I too have had the privilege of providing domiciliary dental care, and during my Dental Foundation training I became concerned about the level of oral care that I saw. I completed a study of the carers working in residential care homes in the area I was working using a questionnaire, to assess how confident they were in providing oral care for their clients and whether they felt further training would be beneficial.

The questionnaire revealed that there is a discrepancy between the levels of training in oral care within different residential care homes. In some centres the carers received no training and in only one centre did all of the carers have training in oral care for their clients. Overall 73.6\% of carers had not received training in caring for their clients' mouths. Without standardised training and monitoring of these standards there is no way of ensuring that residents within care homes are receiving a good standard of oral care.

Although only $26.4 \%$ of the carers had received training, $73.6 \%$ felt confident in providing oral care for their clients. This may be misplaced confidence: as many had not received training they would not have been given a standard of care to reach. Many of the care centres relied upon the carers' knowledge of cleaning their own mouths. This is a flawed strategy in several ways; it assumes that the carers all have good personal oral hygiene on which to base their standards and that there is a similarity in the mouths of the clients and carers. For example, if the client wears dentures but the carer never has, they will be unfamiliar with the techniques and regimes required to maintain a healthy mouth in these clients. Most importantly though it presumes the techniques needed to care for another person's mouth are the same as those needed to care for your own.

Encouragingly, $90 \%$ of the carers that 
completed the study felt that more training would be appropriate. This means that, if training were offered, there should be a high uptake, giving the opportunity to introduce standardised training and monitoring of these standards. With $87.1 \%$ of the carers preferring to have their training in house, this would open the avenue for oral health promotion to be carried out by dental students as part of outreach training, as suggested by A. Korada in the letter Care for all.

Although this study only included care homes within a small area, I feel it may be representative of many areas of Britain and a universal problem needs a universal solution. If training were provided, this would surely improve the standard of living for the elderly and ability impaired in residential care homes.

J. Szuster

Falkirk

DOI: 10.1038/sj.bdj.2009.672

\section{WHAT WERE THEY THINKING?}

Sir, I am glad that the $B D J$ of 25 April 2009 addressed the issue of dental care tourism in the article Travelling for teeth... (BDJ 2009; 206: 425-428).

Most of my practice of 31 years has been with international businessmen and their families who work for global companies and I have had many who opted to have treatment in Hungary and Asia after hearing my estimate and thinking it was 'so expensive.' If I summarise what I saw when they returned, usually with an emergency problem, I can say with surety that periodontal disease was totally ignored. Partially impacted wisdom teeth that trap food interproximally with the second molar and cause massive decay on both teeth are ignored. Almost all root canal treatment is incomplete and periapical abscesses are common; massive cast posts are inserted that fracture roots or make future repair impossible for fear of damaging the remaining root structure; there is massive recurrent decay under many crowns and many crowns have open margins or margins that are totally short of the prepared tooth area. I have seen implants inserted around root remnants from past extractions. I have many patients with multiple splinted crowns that were cast as one unit to save time and effort in places where there seemed to be no rationale for splinting. Most of these splints are totally un-cleansable and caused severe periodontal issues and recurrent decay.

Here is a specific example. I had a husband and wife come for treatment with most existing restorative treatment in total breakdown. The wife accepted my treatment plan and we completed all work on a phased schedule. The husband thought my plan was 'too expensive' and decided to combine his next business trip to Hungary with a dental care vacation. Two years later the wife asked me to see her husband because his teeth were a 'terrible sight.' I saw a new 'round-house' bridge on the upper jaw with most of the porcelain fractured off the metal framework and the remaining porcelain a greenish colour, probably due to the incorrect metal that was used. Many teeth had recurrent decay. Again, he complained that my estimate to repair that damage was too expensive and said he planned a return to Hungary to visit friends and have treatment. A few years later he accompanied his wife to her dental recall visit and told me he did not need a check-up because he had full dentures. I wanted to recommend a cancer check with panoramic radiograph, but felt twice rejected.

I have seen many young international patients in their twenties and thirties with root canal treatment, massive cast posts and splinted crowns on every tooth. They were told that with the new treatment they would not need dental treatment in the future. Then, they came to me for either pain or restorative failures. I had to wonder what were those dentists thinking?! These stories cannot be made up and I have the radiographs and dental records to prove it.

\section{G. Belok \\ New York \\ DOI: 10.1038/sj.bdj.2009.673}

\section{IMPORTANCE OF RADIOGRAPHS}

Sir, I would like to share this case with you. As we all know, remineralisation of enamel can deceive a clinician into underestimating a carious lesion. A 31-year-old patient was seen in my clinic. He presented complaining of discomfort in the region of the lower left wisdom tooth. A full dental and medical history was taken. Examination showed a partially erupted lower left wisdom tooth with an operculum (Fig. 1). My initial diagnosis would have been pericoronitis. The operculum was not erythematous or swollen. I acquired an orthopantomograph on the same day (Fig. 2). To my surprise what looked like a sound tooth showed a significant radiolucency in the coronal region of the

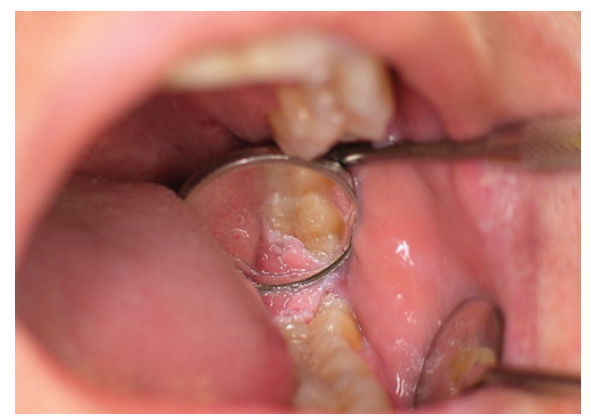

Fig. 1 Partially erupted lower left wisdom tooth with an operculum



Fig. 2 Orthopantomograph

lower left wisdom tooth which extended to the pulp with some periapical radiolucency. The aetiological reason for this patient's complaints was simply caries. In addition there was also caries in the upper left first molar.

This is to show the importance of taking radiographs in the general dental practice as part of the clinical examination.

I note that dentists still refer patients to me without taking radiographs. I could count numerous times when the diagnosis was different to the one the dentist thought, after taking a radiograph.

Y. Zanganah By email DOI: 10.1038/sj.bdj.2009.674

\section{DEMAND FOR NHS PRACTICES}

Sir, it is interesting that amid all the economic doom and gloom there is a Government success story - NHS dentistry. We see hundreds of dental practices in the UK and demand for those with NHS contracts has never been greater. In 
some areas the demand is outstripping the desire for private practices; a situation that many did not predict.

The regular monthly income delivered via the PCT is seen, in the current economic climate, as a much-needed security blanket that ensures the crucial overheads can be covered. For example, we registered more new dentists looking to buy an NHS practice in April 2009 that in the same month in 2008. It naturally follows that the demand for predominantly NHS practices is reflected in goodwill values with practices changing hands for high values.

As we move through the recession and mood lightens it may be that the demand for NHS practices starts to fall away, but for now there is decent demand for NHS contracts with a reasonable UDA value.

\section{A. Acton}

By email

DOI: 10.1038/sj.bdj.2009.675

\section{RANGE OF NORMALITY}

Sir, the letter on aesthetic deviation by S. Radlinskiy (Aesthetic deviation; BDJ 2009; 206: 447) was interesting but based on flawed assumptions.

He says, '...the majority of people have teeth of standard width' and 'standard incisor widths (8.5 $\mathrm{mm})$...'

In an early paper in 1976 I measured approximately 270 extracted central incisor teeth of human origin. The maximum mesio-distal width varied between $7.8 \mathrm{~mm}$ and $10.5 \mathrm{~mm}$ with an average of $9.0 \mathrm{~mm}$. Clearly there is no such thing as a standard width of teeth for the majority of people. As in so many human biological situations there is a range of normality, but few of us are 'standard'.

C. M. Woodhead

By email

DOI: $10.1038 /$ sj.bdj.2009.676

\section{DÉJÀ VU}

Sir, I read the editorial by Mike Mew (BDJ 2009; 206: 393) with interest. Whilst the article is thought provoking, it should have been published as an opinion article rather than an editorial. Leaving much of the conjecture within the article aside, I fail to see the logic in Dr Mew's article that the universities should prove his theories right or wrong. Surely if the philosophy of orthotropics were so effective as to change facial growth, those advocating its use would have amassed a considerable body of scientific evidence to prove its effectiveness by now.

Have we been here before?

G. Mclntyre

Dundee

DOI: 10.1038/sj.bdj.2009.677

\section{CPD TIP}

Sir, I would like to share the following CPD tip:

1. Photograph general CPD docs which you have read (using a mobile phone)

2. Rename the files with the prefix 'CPD $\mathrm{Xh}$ Ym' added to the original file name (eg filename.jpg). The Xh Ym relates to the number of hours and minutes the document represents

3. Upload to your PC

4. Search for 'CPD".*' and transfer them to your CPD folder

5. List the files in date order and count the Xh Ym values for each article to get your current general (nonverifiable figures)

6. Also keep copies of certificates for verifiable CPD.

\section{P. McCrory \\ Manchester \\ DOI: 10.1038/sj.bdj.2009.678}

\section{INSTITUTIONAL FEAR}

Sir, T. Lynn is quite right about the need for the rules governing HIV positive dentists to be changed (BDJ 2009; 208: 508-509). My paper Dentistry and the ethics of infection ( $J$ Med Ethics 2008; 34: 184-187) argues that the current restriction on HIV positive dentists is unethical and seems to have been driven by institutional fear of public fear of infection, rather than any scientific evidence or ethical reasoning. It may be of interest to some of your readers.

$$
\text { DOI: } 10.1038 / \text { sj.bdj.2009.679 }
$$

\section{VALUE FOR MONEY}

Sir, I read with great interest the article entitled Preoperative occlusal matrix aids the development of occlusal contours of posterior occlusal resin composite restorations - clinical rationale and technique by A. Geddes et al. (BDJ 2009; 206: 315-317).
I would like to share my view among your readers regarding the custom made universal occlusal matrix used for the reproduction of the occlusal morphology in posterior composite resin restorations. I have been using this matrix for several years and found it to be very useful. It is flexible and non stick. It compacts and forces the composite into the preparation and approximates the natural anatomy of the existing tooth. They come in different sizes and shapes for molars and premolars of different quadrants which are numbered. It has a handle by which it can be held with tweezers. Once the filling has been cured via the matrix, minimal finishing is required. It saves a lot of time compared to the preoperative occlusal matrix and creates an accurate and aesthetically correct occlusal morphology and pays for itself many times over.

V. Ballal

Manipal

DOI: 10.1038/sj.bdj.2009.680

\section{ROUTINE REFERRAL}

Sir, I enjoyed reading the article Link found between gum disease and heart attack printed in your News section (BDJ 2009; 206: 566).

Now I expect I'm missing something but I understand that some poor folk are genetically susceptible to periodontal disease, and I presume that even if their disease is treated and stabilised, they remain genetically predisposed to periodontal disease. I understand from the article that there is a genetic link between CHD and periodontal disease, but I would presume that this predisposition to CHD would also remain whether the periodontal disease is stabilised or left untreated.

It may be that the presence of some organisms in periodontal pockets might explain the relationship between these two diseases, but the genetic link seems a far more likely explanation.

I wonder if rather than making sure we treat those $10 \%$ or so of periodontally predisposed patients to help prevent CHD we should be routinely referring all those in this susceptible group not just to the periodontist but to the heart specialist as well. DOI: 10.1038/sj.bdj.2009.681 